\title{
Radio observatories and instrumentation used in space weather science and operations
}

Eoin P. Carley ${ }^{1,2, *}$, Carla Baldovin ${ }^{3}$, Pieter Benthem ${ }^{3}$, Mario M. Bisi ${ }^{4}$, Richard A. Fallows ${ }^{3}$, Peter T. Gallagher ${ }^{2,1}$, Michael Olberg ${ }^{5}$, Hanna Rothkaehl ${ }^{6}$, Rene Vermeulen ${ }^{3}$, Nicole Vilmer ${ }^{7,8}$, David Barnes $^{4}$, the LOFAR4SW Consortium ${ }^{3}$

${ }^{1}$ School of Physics, Trinity College Dublin, Dublin 2, Ireland

2 School of Cosmic Physics, Dublin Institute for Advanced Studies, Dublin 2, Ireland

3 ASTRON, Netherlands Institute for Radio Astronomy, Oude Hoogeveensedijk 4, 7991 PD Dwingeloo, The Netherlands

${ }^{4}$ RAL Space, Science \& Technology Facilities Council (STFC) - Rutherford Appleton Laboratory (RAL), Harwell Oxford, OX11 0QX Oxfordshire, UK

5 Department of Space, Earth and Environment, Chalmers University of Technology, Onsala Space Observatory (OSO), 43992 Onsala, Sweden

${ }^{6}$ Space Research Centre of the Polish Academy of Science, 18A Bartycka, 00-716 Warsaw, Poland

${ }^{7}$ LESIA, Observatoire de Paris, Université PSL, CNRS, Sorbonne Université, Universit de Paris, 5 Place Jules Janssen, 92195 Meudon, France

${ }^{8}$ Station de Radioastronomie de Nançay, Observatoire de Paris, PSL Research University, CNRS, Univ. Orléans, 18330 Nançay, France

Received 4 October 2019 / Accepted 23 January 2020

\begin{abstract}
The low frequency array (LOFAR) is a phased array interferometer currently consisting of 13 international stations across Europe and 38 stations surrounding a central hub in the Netherlands. The instrument operates in the frequency range of $\sim 10-240 \mathrm{MHz}$ and is used for a variety of astrophysical science cases. While it is not heliophysics or space weather dedicated, a new project entitled "LOFAR for Space Weather" (LOFAR4SW) aims at designing a system upgrade to allow the entire array to observe the Sun, heliosphere, Earth's ionosphere, and Jupiter throughout its observing window. This will allow the instrument to operate as a space weather observing platform, facilitating both space weather science and operations. Part of this design study aims to survey the existing space weather infrastructure operating at radio frequencies and show how LOFAR4SW can advance the current state-of-the-art in this field. In this paper, we survey radio instrumentation and facilities that currently operate in space weather science and/or operations, including instruments involved in solar, heliospheric, and ionospheric studies. We furthermore include an overview of the major space weather service providers in operation today and the current state-of-the-art in the radio data they use and provide routinely. The aim is to compare LOFAR4SW to the existing radio research infrastructure in space weather and show how it may advance both space weather science and operations in the radio domain in the near future.
\end{abstract}

Keywords: Space weather / Radio

\section{Introduction}

The low frequency array (LOFAR) is a phased array interferometer currently consisting of 13 international stations spread across seven European countries with a central hub of 38 stations in the Netherlands (van Haarlem et al., 2013). The system is operated by the Netherlands Institute for Radio Astronomy (ASTRON), observes in the frequency range of $\sim 10-240 \mathrm{MHz}$, and is used for a variety of astrophysical science use cases, including research into the epoch of reionization

\footnotetext{
*Corresponding author: eoincarley@gmail.com
}

(Patil et al., 2017), pulsar and fast transient observations (Stappers et al., 2011), and large-scale radio surveys (Shimwell et al., 2017), among many others. LOFAR is also involved in solar physics and space weather research, including both quiet and active Sun, heliospheric, and ionospheric observations (e.g., Fallows et al., 2013, 2016; Morosan et al., 2014; Vocks et al., 2018). However, it does not observe the Sun or heliosphere constantly and therefore cannot function as a space weather facility for monitoring or forecasting. A current project known as LOFAR for Space Weather (LOFAR4SW; http:// www.lofar4sw.eu) aims to design an upgrade to LOFAR such that it can observe the Sun, heliosphere, Earth's ionosphere 
and Jupiter simultaneously and constantly throughout its observing window, even while performing observations of other astrophysical objects. Such a system upgrade requires a redesign of both front- and back-end systems, software pipelines redesigns, as well as a redesign of the operational and administrative aspects of the entire system (see Appendix for further details of this upgrade). As part of the design study, a survey was performed to review the current and planned use of radio instrumentation in space weather. The aim of this survey is to define the relationship of LOFAR4SW to the overall landscape of radio space weather research infrastructure. This includes comparing LOFAR4SW to other radio instrumentation, as well as a survey of how radio data are used by space weather service providers.

Instruments operating in the radio domain were some of the first to begin observing space weather phenomena routinely e.g. the early observation of coronal shocks and energetic particles in the form of type II and III radio bursts, respectively (Wild, 1950; Wild et al., 1959). Observations of such phenomena still enjoy a prominent position in space weather science and operations in the modern era, and the variety of radio phenomena under routine observation in a space weather context has grown since these early developments. For example, analysis of solar radio bursts and their relation to flares, coronal mass ejections (CMEs), and solar energetic particles (SEPs) now play a key role in the nowcasting and forecasting of such phenomena (Balch, 1999; Cremades et al., 2015; Zucca et al., 2017); Interplanetary scintillation (IPS) observations of CMEs and the solar wind throughout the heliosphere are becoming a new and integral part of geomagnetic storm forecasting (e.g., Bisi et al., 2010b; Jackson et al., 2015); Observations of ionospheric scintillation, whether due to heliospheric, magnetospheric, or atmospheric drivers, are essential to aid the forecast of radio communication degradations (van de Kamp et al., 2009; Prikryl et al., 2012).

The variety of instruments used to observe these phenomena has also grown, involving both ground- and space-based radio observing infrastructure, covering a frequency range from $\mathrm{kHz}$ to $\mathrm{THz}$ (see Gary, 2016 for a recent solar-specific review). Space weather radio instruments range in complexity from single antennas to long baseline interferometers composed of either dishes or phased arrays. The number of space weather service providers using radio data also continues to grow. These bodies provide daily alerts on space weather conditions to interested stakeholders in industry, such as the aviation, power-grid, and satellite industries. Despite the variety of radio instruments used in space weather and the increasing number of forecasters using radio data routinely, a modern survey of radio instruments and data used in a space weather capacity has not been performed.

This paper aims at such a survey and attempts to define how radio data is used in space weather (for both science and operations), the current instrumentation in existence, which space weather service providers use radio data, the developments and state-of-the-art in space weather radio observations, and finally how a fully built LOFAR4SW fits into this landscape of existing facilities. In Section 1, we describe the primary space weather phenomena and give a brief overview of how radio observations relate to each. In Section 2, we describe the kinds of radio data in use in space weather observations. In Section 3, we survey the instruments currently in use in space weather science and operations. In Section 4, we describe the current major space weather service providers and how/if they use radio data.

\section{Space weather phenomena observed at radio wavelengths}

Radio techniques generally provide access to observations of solar, heliospheric, and ionospheric space weather phenomena. Here we briefly describe the different phenomena and the kind of radio observations that are typically performed of each one.

\subsection{Solar flares}

The sudden release of magnetic energy over tens of minutes results in the acceleration of energetic particles and the emission of light from across the entire electromagnetic spectrum. The flare mechanism can result in both thermal and non-thermal radio emission from a variety of mechanisms including thermal bremsstrahlung (White et al., 2011), (gyro)-synchrotron emission (Dulk \& Marsh, 1982), plasma emission and electron cyclotron maser emission (Melrose, 2017). Exactly which mechanism is observed depends on the conditions of the flaring plasma, and fluxes can range from on the order $10^{4}-10^{10} \mathrm{Jy}$ (where $1 \mathrm{Jy}=1 \mathrm{~W} \mathrm{~m}^{-1} \mathrm{~Hz}^{-1}$ ) depending on the emission mechanism. Typical observations of such activity include monitoring radio flux at discrete frequencies, e.g. with the Radio Solar Telescope Network (Guidice, 1979), observing "solar radio bursts" (SRBs) in dynamic spectra anywhere in the frequency range from $10 \mathrm{kHz}$ to above $1 \mathrm{GHz}$, as well as imaging of the flare-related radio bursts themselves (see Pick \& Vilmer, 2008 for a recent review). Imaging and/or spectroscopy of flare-related radio activity is a powerful diagnostic of flare plasma parameters and electron distribution functions (White et al., 2011), particle acceleration mechanisms (Fleishman et al., 2016), and particle propagation characteristics (Reid \& Kontar, 2018). Hence, these observations are vital for understanding the origin of flare accelerated electrons and the fundamental physics of the flare itself, which are both a major part of space weather research. From an operations perspective, fluxes of solar radio bursts can be so large that they can adversely affect ground and satellite radio communication at Earth (e.g., Marqué et al., 2018), hence there is a need to understand the fundamental flare physics from a radio perspective.

\subsection{Coronal mass ejections}

The release of magnetic energy in the solar corona can also lead to the expulsion of plasma structures known as CMEs. CMEs can travel in excess of the coronal magnetosonic speed and therefore drive shockwaves and accelerate electrons. Plasma emission generated from these electrons is observed as "type II" radio bursts (Nelson \& Melrose, 1985), see Figure 1a and b. They are observed from decimetric to kilometric wavelengths $(\mathrm{kHz}$ to $<1 \mathrm{GHz}$ ), with the long wavelength observation indicating the driving of an interplanetary shock - interplanetary type IIs therefore have been used as a space weather forecasting technique of interplanetary shock arrival at Earth (Warmuth \& Mann, 2004; Cremades et al., 2015). Direct imaging of 

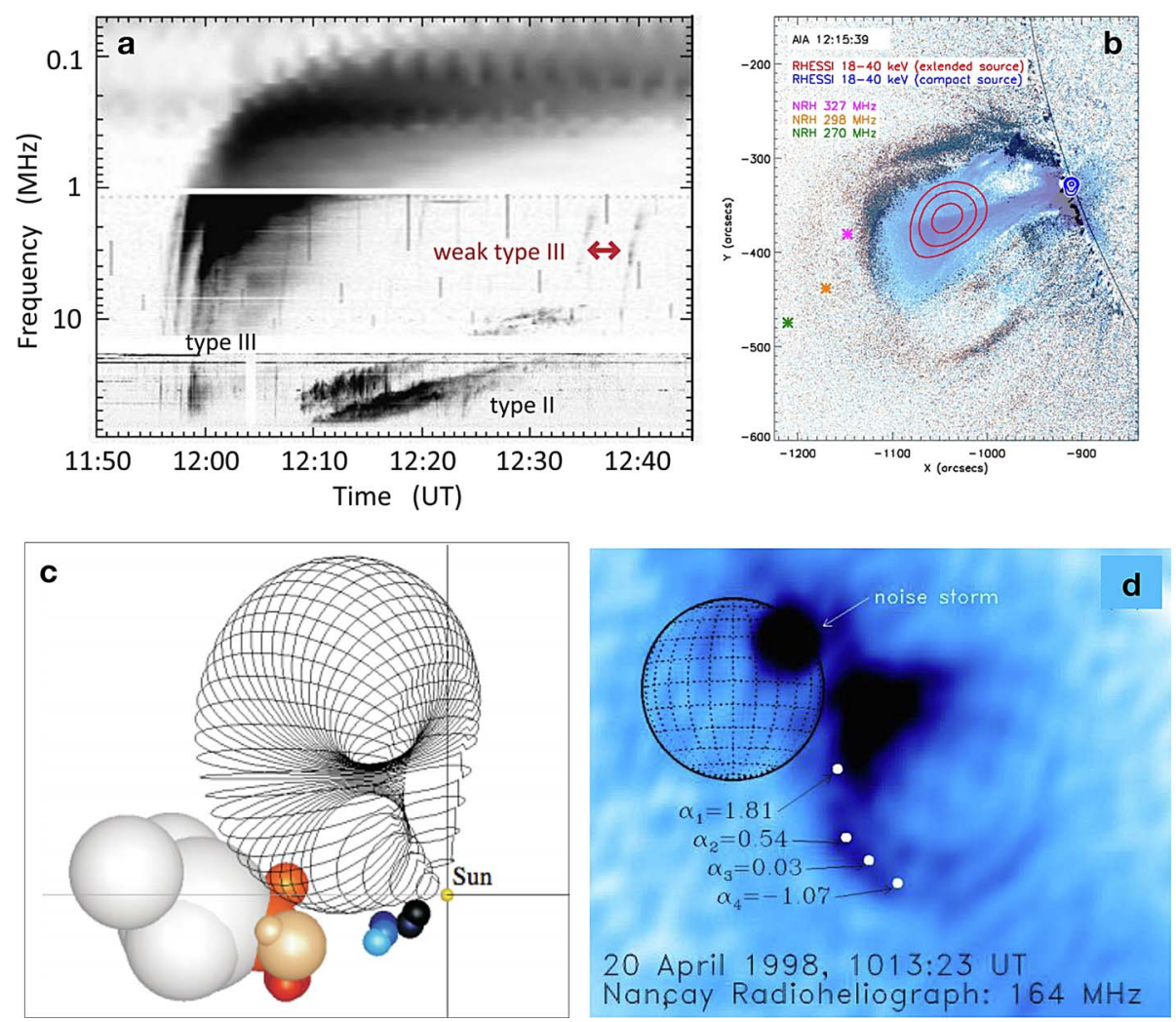

Fig. 1. Examples of solar radio data typically used in space weather science and operations. (a) A dynamic spectrum of solar radio bursts. This is the most typical observation type used to observe flares and eruptive activity in the solar corona. Type IIIs indicate electron beam propagation into the heliosphere, the type II represent the driving of a shock (adapted from Pick et al., 2016). (b) Combination of eruption observation in EUV and type II radio sources (pink, orange, and green points), adapted from Bain et al. (2012). (c) Data-driven modelling of the position of type II and III radio sources with respect to the CME (adapted from Magdalenić et al., 2014). (d) Direct observation of a radio CME using the NRH (Bastian et al., 2001); such observations are rare, but can allow for a diagnostic of CME magnetic field close to the Sun (below a few solar radii)

type II radio sources is typically performed at $\sim 50-450 \mathrm{MHz}$ (Dauphin et al., 2006; Bain et al., 2012; Zucca et al., 2017), which generally provides shock observation below $\sim 3 \mathrm{R}_{\odot}$. Spectral characteristics of type IIs, along with images of their radio sources, can be a good space weather diagnostic of the shock properties in the early phases of eruption, as well as the properties of accelerated electrons themselves (Zucca et al., 2012, 2017; Carley et al., 2013).

While imaging type II shocks is a means of tracking shock activity in the low corona, radio sources from within the CME itself can be observed in the form of type IV radio bursts (these bursts can also have a component associated with the flare, and a sub-class known as "moving type IV" is typically attributed to the $\mathrm{CME}$ ). These radio sources can be used as a rare diagnostic of CME magnetic field in the low corona (Bain et al., 2014; Carley et al., 2017), which is also an important space weather property. They can allow for direct radio imaging of the CME itself (Bastian et al., 2001; Maia et al., 2007) (Fig. 1d), but such observations remain rare. More commonly, type IV bursts are observed as discrete radio sources that are co-spatial with the $\mathrm{CME}$, providing indirect observations of the eruption in the early phases of its evolution, typically below $\sim 3 \mathrm{R}_{\odot}$. Klein et al. (2018) have also recently shown that microwave fluence at the time of CME launch is correlated with CME speed and can hence be a good space weather diagnostic of arrival time at Earth.

One of the few ways to observe a CME in the interplanetary medium is through observations of IPS (e.g., Bisi et al., 2010b). This involves observations of intensity and phase changes of the light from distant radio sources, which can provide a measure of density and indication of magnetic field enhancements in the heliosphere, see Section 4 for details. A primary goal in space weather research and operations is to predict the arrival time of CMEs at Earth, as well as forecasting the strength and direction of the CME magnetic field. Radio techniques remain one of the most promising (if not the only) means of observing these properties remotely before the CME arrives at Earth.

\subsection{Solar energetic particles}

During the flare or CME, energetic particles (electrons, protons, and ionised nuclei of heavy elements) may escape directly into the heliosphere. If detected in-situ they are known as solar energetic particles (SEPs; Reames, 1999). The particles can have energies in the range of $\mathrm{keV}$ to $\mathrm{GeV}$ in the largest events. Both the electrons and heavier nuclei can affect spacecraft electronics (Xapsos et al., 2007; Glover et al., 2008), while the protons and heavier nuclei pose a radiation hazard to 
astronauts or crew of trans-polar flights, with the hazard depending on SEP intensity and energy (Cucinotta et al., 2010; Reames, 2013). Radio spectroscopic signatures in the form of type IIIs provide insight into SEP release into the heliosphere (Klein et al., 2010; Kouloumvakos et al., 2015). The details of the spectral characteristics and imaging of type IIIs can be used as an indicator of SEP release time and position, as well as a diagnostic of their means of propagation throughout the heliosphere (at least for the electrons) (Agueda et al., 2014). Despite efforts in forecasting SEPs, which sometimes involve solar radio bursts (Balch, 1999, Zucca et al., 2017), SEP arrival time prediction remains one of the most difficult aspects of space weather. Much of the difficulty is in determining SEP origin (either flare or shock see e.g., Kahler, 2007), and determining the complicated particle injection and transport physics of SEPs through the heliosphere (e.g., Agueda \& Lario, 2016; Lario et al., 2017; Dresing et al., 2018; Laitinen et al., 2018).

\subsection{Solar wind}

The constant outflow of plasma from corona to heliosphere is known as the solar wind, which generally comes in two varieties; the slow wind with an average speed of $\sim 350 \mathrm{~km} \mathrm{~s}^{-1}$ and fast wind with average speed of $\sim 750 \mathrm{~km} \mathrm{~s}^{-1}$ at $1 \mathrm{AU}$. The slow wind is thought to emerge from closed regions of magnetic field in the corona, while the fast wind emerges from regions of open magnetic field known as coronal holes (Cranmer, 2009). Since coronal holes can be maintained for months and rotate roughly at the same rate as the Sun, conduits of fast wind that impact on neighbouring slow wind streams can be maintained for months. Such regions of interacting fast and slow wind constantly sweep around the solar system and are known as co-rotating interaction regions (CIRs). If a CIR impacts the Earth, it can cause a geomagnetic disturbance known as a sub-storm (Tsurutani et al., 2006). Tiburzi et al. (2019) has recently shown the effectiveness of using dispersion measures of low frequency radio observations of pulsars in characterising the state of the solar wind, while Bisi et al. (2010a) and Richardson (2018) have also shown that similar techniques in interplanetary scintillation can be used to observe CIRs well inside Earth-orbit.

\section{5 lonospheric disturbances}

Conditions in the ionosphere can become disturbed due to the increased levels of X-ray and EUV emission during solar flares, leading to increases in the total electron content (TEC) in the ionosphere (e.g., Selvakumaran et al., 2015). Furthermore, significant levels of geomagnetic activity caused by disturbances in the solar wind from CIRs or CMEs can lead to increased activity in the ionosphere, e.g. sudden ionospheric disturbances, increased turbulence, a variety of ionospheric currents, particle instabilities, and waves in ionospheric plasma (Tsurutani et al., 2009). Plasma turbulence in the ionosphere can strongly affect many of the technologies upon which we increasingly rely, including satellite communications, radar target detection, and precision navigation via Global Navigation Satellite Systems (GNSS). Use of radar, scintillation measures, and spectroscopic signatures of such activity (see Sect. 4) can aid the diagnostics of ionospheric disturbances and serve as a measure of radio communication quality during times of elevated solar activity (Žigman et al., 2007; Yasyukevich et al., 2018)

\subsection{Geomagnetic storms}

Impact of CIRs or CMEs on the Earth's magnetospheric environment can lead to a highly disturbed geomagnetic field, known as a geomagnetic storm (Kappenman, 1996). Geomagnetic storms are most powerful when the interplanetary magnetic field is oppositely directed to the Earth's geomagnetic field i.e. when the interplanetary " $B_{z}$ component" is directed southward. This causes increased levels of magnetic reconnection on the day-side geomagnetic field and increased currents in the ionosphere, ultimately leading to geomagnetically induced currents (GICs) in ground-based technologies like electricity grids or oil and gas pipelines (e.g., Boteler et al., 1998; Trichtchenko \& Boteler, 2001). Hence, predicting geomagnetic storms is an essential aspect of space weather monitoring. To date, such forecasts have proved the most challenging aspect of space weather science and prediction. Radio observations of IPS and Faraday rotation (see Sect. 4) remain the only viable method for remote monitoring of the strength and direction of the interplanetary magnetic field (Bisi et al., 2010b) and hence one of the few methods of forecasting these properties.

\section{Space weather impacts and indices; radio perspective}

Each of the space weather phenomena described above is an active area of space weather research from a scientific perspective. As described, these phenomena can have an impact on Earth's technological infrastructure, and these impacts are generally measured via space weather indices. These indices are metrics used to define the current space weather conditions in an operational capacity, and radio observation can contribute to their forecast and nowcast in a variety of ways.

\subsection{Indices of geomagnetic storms}

The severity of geomagnetic storms (and subsequent GICs) is monitored via a large number of worldwide magnetometers (e.g. Wei et al., 2013). Measurement of the components of the Earth's electromagnetic field over time can be used to derive indices that indicate the current state of the geomagnetic field or severity of any geomagnetic storm, including the Dst-index (a measure of the increase of ionospheric ring current), $k$-index (a measure of the regional or local rate of change of the geomagnetic field), and $k p$-index (a planetary-scale version of $k$-index), and NOAA's G1 (minor) to G5 (severe) rating. Radio observations may indirectly contribute to the forecast of these indices via observations of IPS. However, forecasting the severity of a geomagnetic storm (Dst and $k p$-strength) from the properties of an earth-bound CME or solar wind transient remains a significant research challenge. There is currently no known way to remotely observe interplanetary magnetic field strength and direction, but recent research has shown that radio diagnostics of the Faraday rotation of light from radio sources passing through the heliosphere could provide insight into these properties (see Sect. 4). Hence the radio domain is one of the primary candidates for accurate geomagnetic storm prediction in the near future. 


\subsection{Indices of radio communication interruption}

The ionosphere can become disturbed during times of increased solar activity, resulting in interruptions in the quality of radio communications. There are a variety of measures of the quality of ground- and spaced based radio communications via the ionosphere. These include the $\mathrm{S} 4, \phi$ indices - a measure of the variation in intensity and phase of GNSS signals at $1.58 \mathrm{GHz}$ and $1.23 \mathrm{GHz}$ coming through the ionosphere (the technique can be applied to many other frequencies but GNSS carrier frequencies are the most widely used). The total electron content (TEC) of the ionosphere is an important parameter related to the frequency of radio waves which experience transmission or reflection from the ionosphere and is derived from GNSS, ionosonde or active/passive radar (see later). Related to this, the maximum usable frequency (MUF) is the highest radio frequency that can be used for transmission between two points via reflection from the ionosphere. Other parameters include the highest ordinary-wave frequency (fofE) reflected back from a sporadic $\mathrm{E}$ layer, the altitude of the peak density in the ionospheric F2 layer ( $\mathrm{hmF} 2)$, and the plasma frequency of the F2 layer (foF2), each observed by ionosondes (see Bilitza et al., 2014 for a review of the International Reference Ionosphere model in relation to these indices). The variability of flux at $10.7 \mathrm{~cm}$ (F10.7) is also used as a proxy for full Sun EUV flux and can be used as an input into ionospheric models. There has also been recent suggestion of the use of F11.1 as a compliment to this (Acebal \& Sojka, 2011). Each of the above metrics are constantly monitored worldwide as a means of nowcasting global radio communication quality through the ionosphere and they are some of the most popular metrics of space weather activity observed at radio frequencies.

In addition to disturbed radio communications due to ionospheric conditions, radio communications can also be rendered ineffective due to high flux from solar radio bursts. For example, the high intensity of solar radio bursts (sometimes greater than $10^{5}$ solar flux units $(\mathrm{SFU})$, where $1 \mathrm{SFU}=$ $10^{4} \mathrm{Jy}=10^{-22} \mathrm{~W} \mathrm{~m}^{-2} \mathrm{~Hz}^{-1}$ ) can result in carrier-to-noise ratio degradation in the L1 observing channel of GPS satellites (Cerruti et al., 2006). In extreme cases the interference can be so bad it may also result in loss of GPS navigational lock at sites across the Sun-lit side of the Earth for up to 10 min (Cerruti et al., 2008). Recent results have shown that such a degradation in GPS signals can result in position errors of up to $300 \mathrm{~m}$ (Muhammad et al., 2015). Marqué et al. (2018) has also shown recently that communications for aeronautical navigation at $\sim 1 \mathrm{GHz}$ may also experience interference during intense solar radio bursts. There is no universal metric of radio burst intensities, but one standard is the National Oceanic and Atmospheric Administration (NOAA) Space weather Prediction Center (SWPC) R1-R5 rating, rated minor to extreme in terms of radio burst flux.

\subsection{Radiation hazards}

The arrival of SEPs at Earth can cause damage to satellite electronics (via either electronic discharge or heavy-ion impact on microelectronics) and cause a radiation hazard for astronauts or the crew of high-latitude flights, with the level of risk depending on particle intensity and energy (Glover et al., 2008; Cucinotta et al., 2010; Reames, 2013). The indices by which particle radiation storms are measured are varied, but one of the primary metrics is the NOAA SWPC S1-S5 index. Radio observations are sometimes involved in nowcasting of SEPs (Balch, 1999; Zucca et al., 2017), and could potentially be used to nowcast the severity of particle radiation storms. However, the impact of SEPs remains a significant challenge in both space weather nowcasting and forecasting (Anastasiadis et al., 2017).

\section{Radio observation techniques used in space weather}

There are a variety of techniques used routinely in radio observations of space weather phenomena. These include some well known techniques such as monitoring total solar flux with time series, dynamic spectroscopy of solar radio bursts, and interferometric imaging. Some typical examples of these observation types are shown in Figure 1 and the instruments that observe these data types are outlined below. In this section, we outline some of the latest techniques available for radio space weather observations, particularly those used by phasedarray interferometers such as LOFAR. The additional advantage of a phased array is the ability to beam-form in potentially hundreds of different directions at once (van Haarlem et al., 2013). This allows for the observation of hundreds of sources on the sky and in the case of LOFAR4SW gives the ability to simultaneously observe the Sun, heliosphere, and ionosphere (as well as any other astrophysical observation). The latest space weather techniques which can generally make use of beamforming are as follows.

\subsection{Tied-array solar imaging}

Phased array instruments such as LOFAR can produce multiple telescope beams on the sky in the same or many different directions. This effectively allows for observation of time series (and dynamic spectra) along multiple lines-of-sight. When these multiple beams are arranged in a grid-pattern in a single direction (at a single source) on the sky, the technique is known as "tied-array imaging", see Figure 2 for an example applied to solar observations using LOFAR. In recent years such a technique has been successfully employed to perform spatially resolved observations of solar radio bursts (e.g., Morosan et al., 2014, 2015; Reid \& Kontar, 2017). Compared to interferometric imaging, the advantage of such a technique in the case of LOFAR is its much higher time-sampling, e.g. the time resolution of tied-array imaging with LOFAR is potentially as high as micro-seconds (compared to $>0.1 \mathrm{~s}$ for interferometric imaging), depending on the frequency resolution. Such high time resolution has provided a means of observing the origin of fine structure and how radio waves propagate through the corona (Kontar et al., 2017). Furthermore, one of the advantages of the LOFAR system is that interferometric and tied-array imaging can also be performed simultaneously.

\subsection{Observations of interplanetary scintillation}

While beam forming on the Sun has lead to breakthroughs in the understanding of solar radio bursts, beam-formed 


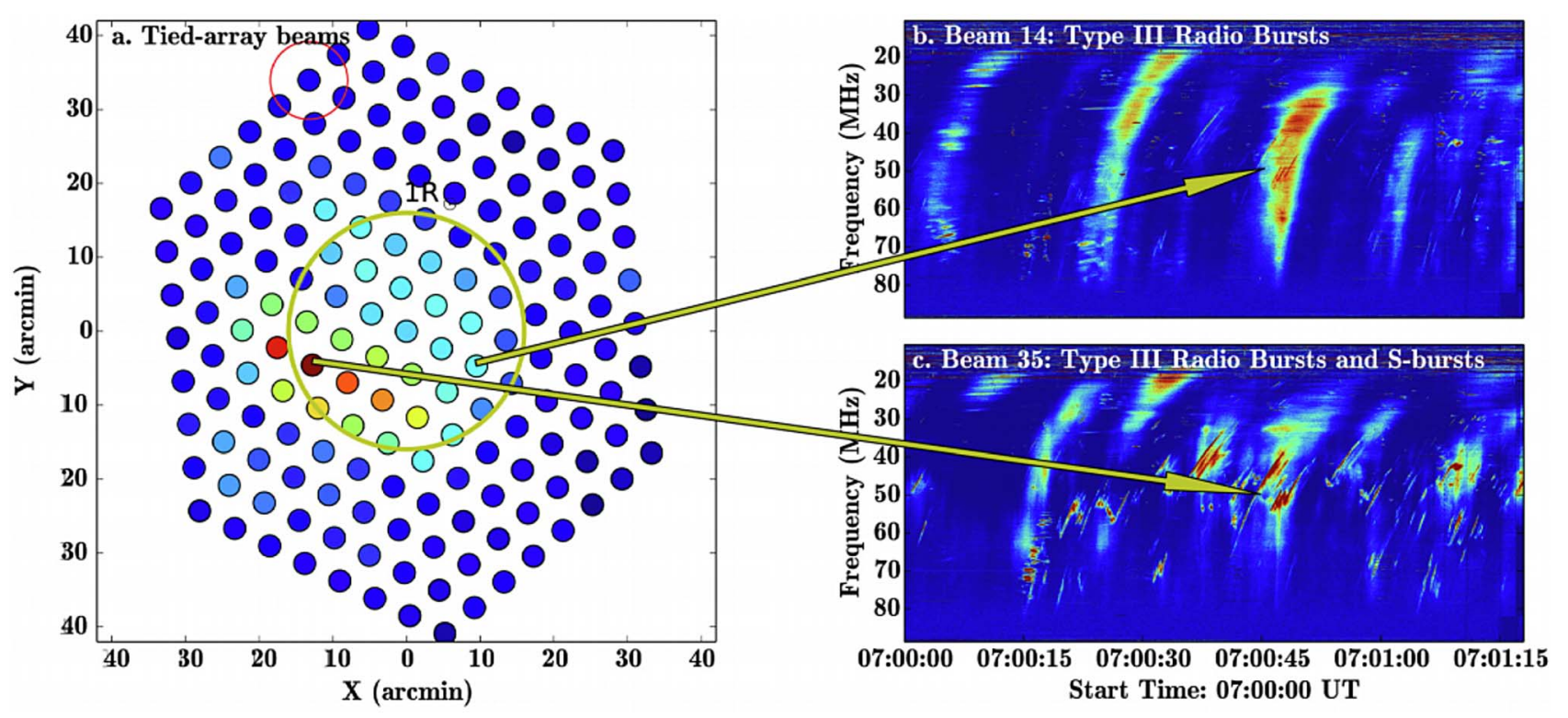

Fig. 2. An example of tied-array imaging applied to the solar corona from Morosan et al. (2015). Using LOFAR core stations, up to 488 beamlets can be arrange in a grid over the Sun, each giving a dynamic spectrum of the radio activity at that particular location. This allows for spatially resolved observations of radio bursts on the Sun, in this case the examination of the origin of fine structure known as S-bursts.

observations of distant radio sources are used to perform observations of IPS. This technique monitors the variability in flux and phase of distant radio sources and can be used to diagnose density and velocity variations (from CIRs/SIRs or CMEs) in the interplanetary medium, see Figure 3. Observations of IPS can be used to monitor and sometimes forecast solar wind/CME conditions (Jackson et al., 2013) and the technique has been demonstrated using multiple instruments of the Worldwide IPS Stations (WIPSS) network. If there is full Stokes polarisation information available then it is theoretically possible to estimate the solar wind/CME magnetic field strength and direction via monitoring of a phenomenon known as Faraday rotation (e.g., Jensen et al., 2010). As light from a distant radio source travels through the interplanetary medium (IPM), the plane of polarisation of the radio waves rotate, with the amount of rotation depending on the magnetic field strength and direction in the IPM or any CME contained within it. This can lead to a calculation of the line-of-sight magnetic field component of a CME in interplanetary space, and some promising results have recently been shown using the Very Large Array at 1-2 GHz for a CME at 6-15 $R_{e}$ (Kooi et al., 2017). However, observing CMEs further into the heliosphere (0.4 AU) at LOFAR frequencies must contend with the additional Faraday rotation also experienced by emission passing through the ionosphere, so distinguishing the rotation component due to a CME alone remains a significant challenge in radio space weather studies. Nonetheless, efforts are underway to achieve this and LOFAR4SW aims to be a pioneer in this effort in the near future.

One of the most advanced space weather forecasting techniques is in the use of magnetohydrodynamic (MHD) models of the solar wind (including transients) throughout the heliosphere, such as the ENLIL model (e.g., Odstrcil, 2003). ENLIL (named after the Sumerian "God of Wind") is a large-scale, MHD-based numerical model of the heliosphere, used to provide 1-4 day advance warning of solar wind structures and Earth-bound CMEs. The model requires input of the boundary conditions in the solar wind at $\sim 21 R_{\odot}$, and density and velocity observations of IPS are now used routinely to provide these boundary conditions (Jackson et al., 2015; Yu et al., 2015). IPS + ENLIL is now used by multiple space weather service providers to provide forecasting of solar wind plasma parameters days in advance, see Section 5.2.

\section{3 lonospheric scintillation and active/passive radar}

Beam-forming can also be used to monitor ionospheric conditions in both space and time. Ionospheric studies in a space weather context primarily use radar techniques that are classified as a subset of two categories, namely active and passive radar. Active radar consists of transmitting a radio signal into the ionosphere and receiving the reflected/returned signal with a receiver. Comparing transmitted and returning signals provides an analysis of ionospheric conditions and hence provides useful information of the quality of radio communications that interact with the ionosphere. Passive radars do not transmit, but receive signals from known transmitters, such as TV and radio transmission channels. The techniques are varied but mainly consist of:

- Ionosonde (active): Ionospheric sounders use radio transmissions that experience total reflection at the local plasma frequency. From the analysis of the reflected signal, electron density characteristics of the ionosphere (TEC maps) can be derived. Different frequencies can probe different altitudes/regions in the ionosphere, and they primarily operate in the HF range, see Figure 4.

- Riometry (passive): Relative Ionospheric Opacity Meters for Extra-Terrestrial Emissions of Radio waves measure the absorbed power from galactic sky noise, which is a 

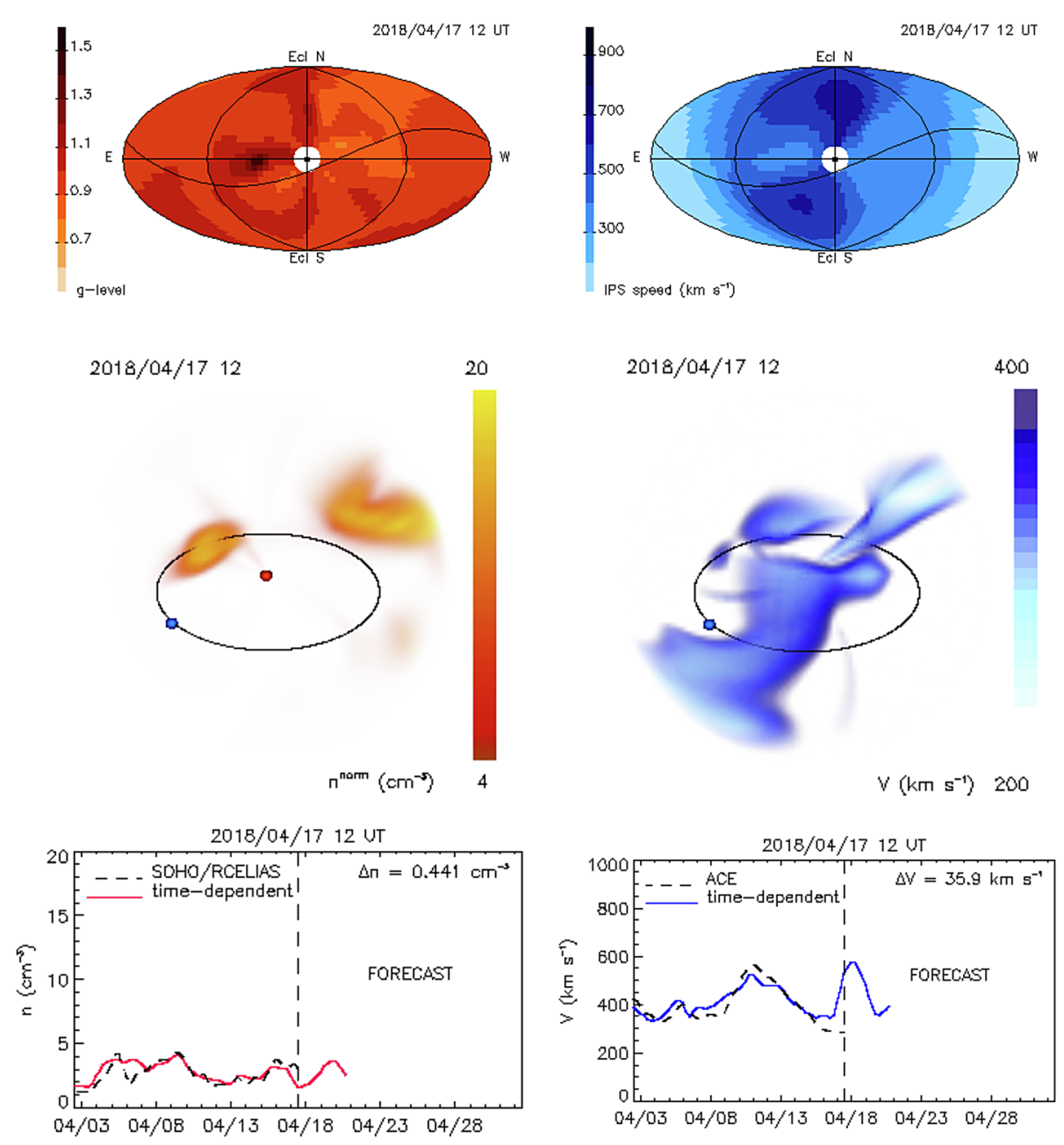

Fig. 3. Examples of heliospheric observations from IPS typically used in space weather science and operations. (a) IPS g-level (related to density fluctuation in the interplanetary medium) and speed. (b) Tomographic 3-D reconstruction of properties in top panel. (c) SOHO data of velocity of solar wind (left) and ACE data of density (right) with respect to time, plotted with forecast of the same property using observations of IPS. Observations such as these are now used routinely in space weather forecasting. Realtime data are available at http://ips.ucsd.edu/.

function of the degree of ionisation (electron density) of the ionospheric $\mathrm{D}$ region $(70-100 \mathrm{~km}$ altitude). LOFAR4SW intends to operate as a passive system for ionospheric studies monitoring, including riometry.

- Incoherent scatter radar (active): A technique that observes weak scatter from thermal plasma ion-acoustic and Langmuir mode resonant oscillations. Primary measurements include electron density, electron temperature, ion temperature, and ionospheric drifts. Operation can be in HF/VHF/UHF/L-band.

- Coherent scatter radar (active): These systems measure coherent scatter from ionospheric irregularities due to plasma instabilities, waves, and structures. Modern systems perform radar imaging, and can operate in the HF domain.

LOFAR4SW will be able to perform routine observations of the ionosphere using passive radar techniques that monitor ionospheric disturbances. It will also monitor scintillation in the ionosphere in much the same way as GNSS receivers. GNSS antennas receive the signals from satellite beacons and monitor the amplitude and phase change of discrete single-frequency time-series', providing information on the S4-index and $\phi$-index, and conditions in the ionosphere such as the total electron content (TEC), among other properties (e.g., Vilà-Valls et al., 2017). However, such measurements are usually performed at a single frequency and spatial location; much greater information can be found in the dynamic spectrum over a wide frequency bandwidth (e.g., Fallows et al., 2014), and making use of the wide geographic distribution of LOFAR4SW stations across Europe. Widespread stations and the ability to beamform towards multiple points on the sky will enable the dynamics of large-scale phenomena, such as Travelling Ionospheric Disturbances (TIDs), to be studied, while small-scale dynamics can be viewed using the dense LOFAR core stations.

The current operational plan for LOFAR4SW is the performance of daily observations using solar imaging spectroscopy, solar tied-array imaging, IPS, FR, and ionospheric studies using both passive radar and scintillation techniques. Each of these techniques will be performed simultaneously, making LOFAR4SW one of the few instruments in the world capable 


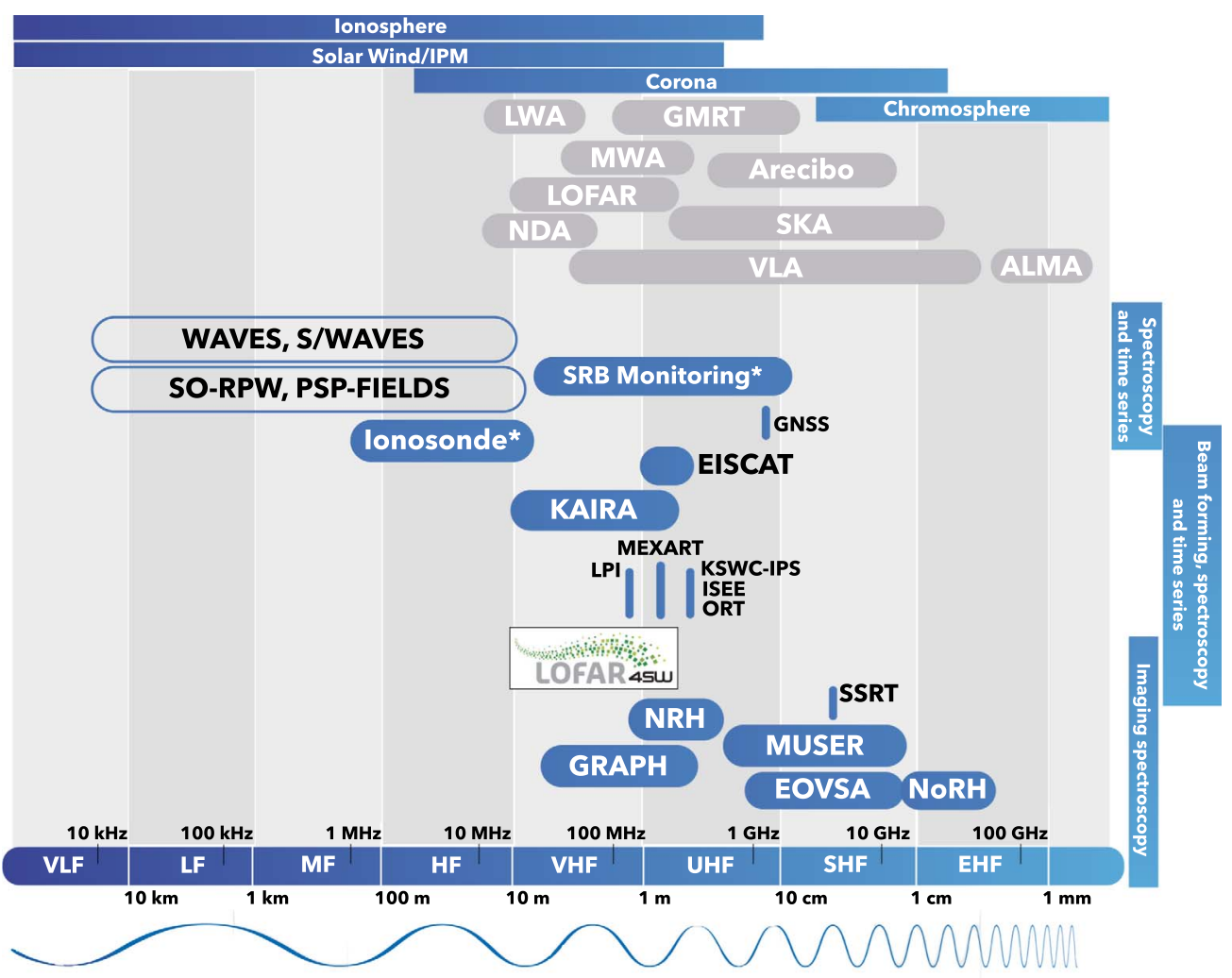

Fig. 4. A comparison of LOFAR4SW to other solar physics or space weather radio instruments. The bottom edge indicates frequency, wavelength, and International Telecommunication Union (ITU) designated name of each frequency band. The top of the graphic indicates the heliospheric domain that such frequencies generally give observational access to. The graphic on the right indicates the observational technique that the instruments use. The bubble for each instrument indicates its frequency range. Instruments in blue are solar/heliosphere/ionosphere dedicated, while those in grey can observe the Sun but are not (will not be) dedicated instruments. Specific details of each instrument are given in Table 1 .

of simultaneous observation of the Sun, heliosphere, and ionosphere in a space weather science and operations capacity.

\section{Space weather radio research infrastructure}

This section provides information on space weather research infrastructures, which include the instruments used to perform space weather observations and the bodies that use the data from these instruments to provide space weather forecasts, warnings and other services. While regional surveys of such infrastructures exist (see Denardini et al., 2016 for a recent review of space weather research networks in Latin America), our survey concentrates on the worldwide infrastructure, specifically operating in the radio domain.

\subsection{Space weather instruments operating at radio wavelengths}

In the following, a space weather instrument is any groundor space-based instrument that is capable of monitoring solar, heliospheric and/or ionospheric phenomena, enabling both space weather science, and forecasting. There are a huge number of instruments operating in the radio domain, ranging in complexity from small GNSS antennas to long-baseline phased-array interferometers such as LOFAR. Such instruments observe one or more of the phenomena outlined in Section 2 and are either dedicated solar instruments (therefore offering some level of space weather monitoring) or non-dedicated (contributing to Space Weather Science). Here we mainly concentrate on instruments that are dedicated to observing the Sun, heliosphere, and/or ionosphere. Figure 4 attempts to summarise the primary instruments performing radio space weather science and/or operations. It shows instruments operating from frequencies of $\mathrm{kHz}-\mathrm{THz}$, the heliospheric domain such frequencies generally give access to, and the type of observations made (imaging spectroscopy, beam forming and spectroscopy, or time series and spectroscopy). Those instruments shown in grey can observe solar and space weather phenomena, but are not dedicated instruments.

For solar observations, there are a limited number of interferometers that observe the Sun routinely, including the Nançay Radioheliograph (NRH; Kerdraon \& Delouis, 1997), located in central France and observing the solar corona at ten discrete frequencies from $150 \mathrm{MHz}$ to $450 \mathrm{MHz}$; the recently built Mingantu Solar Radioheliograph (MUSER; Mei et al., 2018) located in inner Mongolia and performing imaging spectroscopy of the solar corona from $0.4 \mathrm{GHz}$ to $15 \mathrm{GHz}$; the Gauribidanur 


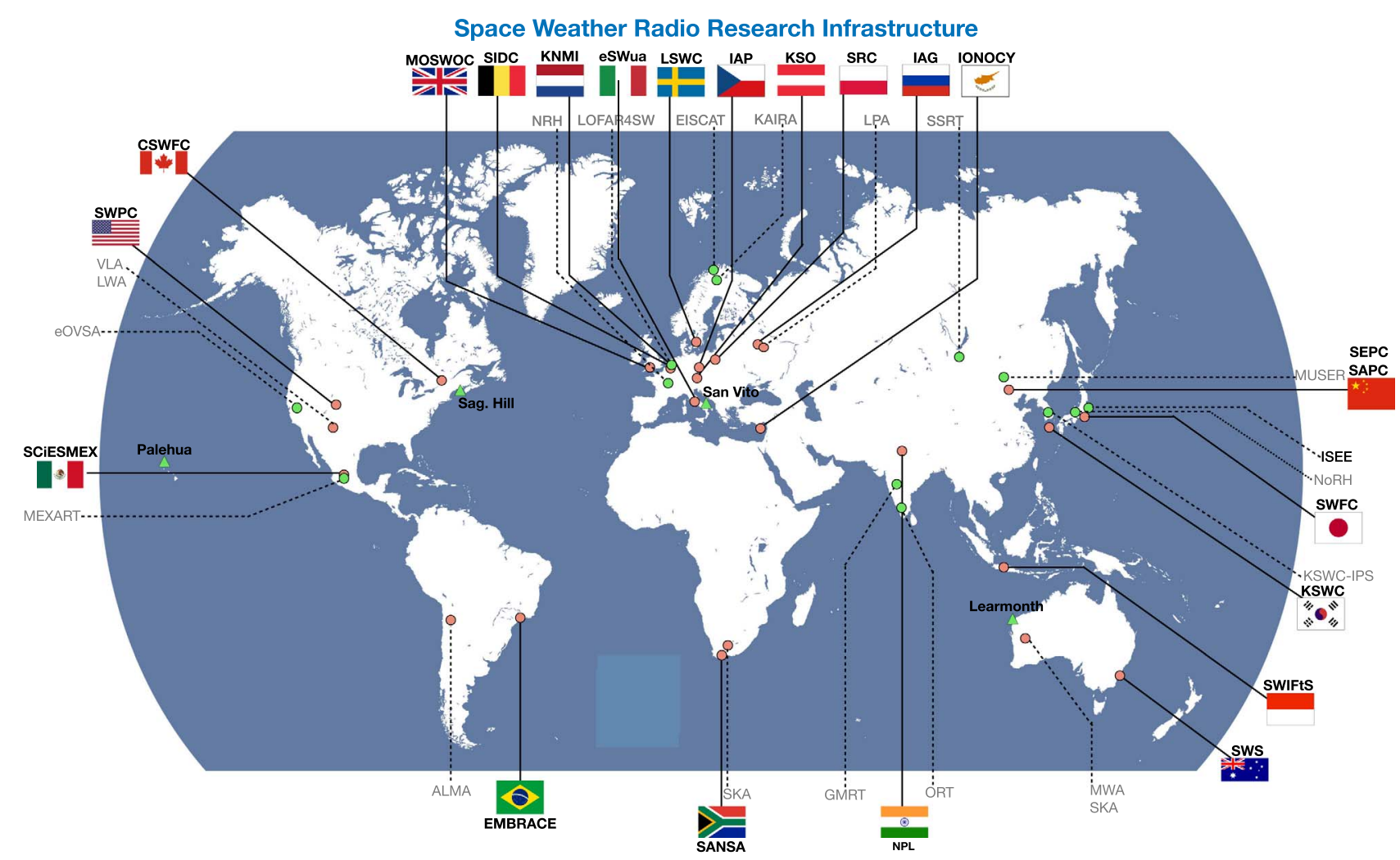

Fig. 5. Worldwide distribution of space weather service providers (indicated by national map and described in Table 1) and the primary instruments offering radio observations of space weather phenomena (outlined in Fig. 4 and Table 1). Green circles indicate the instruments that are space-weather dedicated, with orange circles indicating instruments that can perform solar observations but don't do so routinely. Green triangles indicate the positions of the radio spectrometers of RSTN which provide routine SRB monitoring and alerts.

Radioheliograph (GRAPH; Ramesh et al., 1998) in southern India, operating at discrete frequencies between $40 \mathrm{MHz}$ and $150 \mathrm{MHz}$; the Expanded Owens Valley Solar Array (EOVSA; Kuroda et al., 2018) in the USA, performing imaging spectroscopy from $1 \mathrm{GHz}$ to $18 \mathrm{GHz}$; the Japanese Nobeyama Radioheliograph (NoRH; Nakajima et al., 1994) operating at $17 \mathrm{GHz}$ and $34 \mathrm{GHz}$, and the Siberian Solar Radio Telescope (SSRT; Grechnev et al., 2003) operating at the single frequency of $5.7 \mathrm{GHz}$ (the location of these and other instruments are shown in Fig. 5). Of the numerous interferometers performing imaging spectroscopy of the Sun routinely, LOFAR4SW will have the lowest operating frequency, down to the ionospheric cut-off (imaging to the greatest altitude in the corona of $\sim 3 R_{\odot}$ ). The lower frequency bands observed by LOFAR (4SW) are vital observations, giving access to regions of the corona where eruptions are accelerated, shocks are driven, and energetic particles are released into the heliosphere. Such observations are therefore essential for understanding earlyphase space weather phenomena. LOFAR4SW is also unique in the sense that it is one of the few instruments capable of imaging spectroscopy both as an interferometer and as a tiedarray beamformer. It can also perform these modes simultaneously, providing the dual advantage of high sensitivity and spatial resolution of interferometry and high temporal resolution (potentially mili or microseconds) of tied-array imaging. In this sense, it will be the most diverse amongst the imaging spectrometers that observe the Sun on a routine basis.
Since the LOFAR4SW system will act as an interferometer and a beam-former, it will also bridge the gap between instruments that are solely used to image the Sun (e.g., NRH and MUSER) and those that perform observations of IPS in the heliosphere, including the Big Scanning Array of Lebedev Physical Institute (BSA-LPI; Dagkesamanskii, 2009) operating at $111 \mathrm{MHz}$ and located in Russia; the Mexican Array Radio Telescope (MEXART; Mejia-Ambriz et al., 2010) operating at $140 \mathrm{MHz}$; the Korean Space Weather Centre (KSWC) IPS array (http://spaceweather.rra.go.kr) operating at $327 \mathrm{MHz}$; the Solar Terrestrial Environment Laboratory ISEE IPS array operating in several locations in Japan at $327 \mathrm{MHz}$ (Asai et al., 1995); and the Ooty Radio Telescope (ORT; Sukumar et al., 1988) also operating at $327 \mathrm{MHz}$, located in India. Observations of IPS are also part of the wider plans for space weather monitoring as part of the the Chinese Meridian Project (Wang, 2010).

Of those instruments that observe the heliosphere through IPS, LOFAR4SW will be one of the few instruments to do so over a broad frequency band, as opposed to the single frequency phased arrays that are commonly used, and with the ability to observe multiple sources simultaneously. The IPS-capable stations listed above are officially grouped into a consortium known as the Worldwide Interplanetary Scintillation Stations (WIPSS; Bisi et al., 2017) network, the goal of which is to routinely provide IPS observations for space weather science and forecasting purposes. 
E.P. Carley et al.: J. Space Weather Space Clim. 2020, 10, 7

Table 1. Radio instruments performing observations for space weather science and/or operations.

\begin{tabular}{|c|c|c|c|c|c|c|}
\hline Instrument & $\begin{array}{l}\text { Frequency } \\
(\mathrm{MHz})\end{array}$ & $\begin{array}{l}\text { Local noon } \\
\text { (UTC) }^{\mathrm{a}}\end{array}$ & Observable & $\begin{array}{l}\text { Angular } \\
\text { resolution }\end{array}$ & $\begin{array}{l}\text { Operational } \\
\text { phase }\end{array}$ & $\begin{array}{c}\text { SW } \\
\text { monitoring }^{\mathrm{c}}\end{array}$ \\
\hline LOFAR & $10-240$ & 11 & Sun, IPM, Iono & $1.4^{\prime}-30^{\prime \prime}$ (core stations) & $>2006$ & No \\
\hline LOFAR4SW & $10-240$ & 11 & Sun, IPM, Iono & $1.4^{\prime}-30^{\prime \prime}$ (core stations) & $>2023$ & Yes \\
\hline SKA & $350-14000$ & 10 (SA), 4 (AUS) & Sun, IPM, Iono & TBD & $>2020$ & No \\
\hline MUSER & $400-15000$ & 4 & Sun & $1.3^{\prime}-50^{\prime \prime}$ & $>2014$ & Yes \\
\hline FAST & $70-3000$ & 4 & Sun & $0.8^{\prime}-30^{\prime}$ & $>2017$ & No \\
\hline EOVSA & $1000-18000$ & 20 & Sun & $0.05^{\prime}-1^{\prime}$ & $>2015$ & Yes \\
\hline GRAPH & $50-150$ & 7 & Sun & $3^{\prime}-11.2^{\prime}$ & 1997 - present & Yes \\
\hline VLA & $74-50000$ & 18 & Sun & $1.2^{\prime \prime}-13^{\prime}$ & $>1980$ & No \\
\hline OVRO-LWA & $10-88$ & 18 & Sun, IPM, Iono & $2-20^{\circ}$ & $>2009$ & Yes $^{\mathrm{d}}$ \\
\hline NoRH & 17,34 & 3 & Sun & $0.07^{\prime}-0.1^{\prime}$ & $>1992$ & Yes \\
\hline SSRT & 5700 & 5 & Sun & $15^{\prime \prime}$ & >1996 & Yes \\
\hline EISCAT 3D & $218-248$ & TBD & IPM, Iono & TBD & $>2021$ & TBD \\
\hline KSWC-IPS & 327 & 4 & IPM & N/A & N/A & Yes \\
\hline Ionosondes ${ }^{b}$ & $0.5-20$ & $0-24$ & Iono & N/A & N/A & Yes \\
\hline ISEE & 327 & 3 & IPM & N/A & $>1980 \mathrm{~s}$ & Yes \\
\hline KAIRA & $10-240$ & 11 & Iono & $2-20^{\circ}$ & $>2012$ & Yes \\
\hline RSTN & $25-85$ & $0-24$ & Sun & N/A & $>1980 \mathrm{~s}$ & Yes \\
\hline WIND/WAVES & $0.01-14$ & $0-24$ & Sun, IPM & N/A & $>1996$ & Yes \\
\hline STEREO/WAVES & $0.01-16$ & $0-24$ & Sun, IPM & N/A & 2006-2015 & Yes \\
\hline SO RPW & $0.01-20$ & Intermittent & Sun, IPM & N/A & 2019-2022 & Yes \\
\hline PSP FIELDS & $0.01-20$ & Intermittent & Sun, IPM & N/A & 2019-2025 & Yes \\
\hline $\mathrm{SRB}$ monitoring ${ }^{\mathrm{b}}$ & $10-5000$ & $0-24$ & Sun & N/A & N/A & Yes \\
\hline
\end{tabular}

${ }^{a}$ If the observatory performs daytime space weather observations it may do so at any time when the Sun is visible, however nominal operation is likely to be several hours centered on local noon.

$\mathrm{b}$ There are hundreds of global ionosondes and SRB monitoring observatories. The parameters presented here are a generic representation of their nominal operational ranges.

${ }^{c}$ SW monitoring refers to whether or not the instrument observe the sun, heliosphere or ionopshere constantly throughout its observing window.

d OVRO-LWA is currently being upgraded with a solar-dedicated backend for space-weather-capable imaging spectroscopy (completion expected in $\sim 2$ years).

LOFAR4SW will routinely monitor the ionosphere and will provide complimentary observations alongside the Kilpisjärvi Atmospheric Imaging Receiver Array (KAIRA; McKayBukowski et al., 2015), which is an independent station using LOFAR hardware, located in northern Finland and dedicated to observations of the ionosphere through passive radar; the European Incoherent Scatter (EISCAT; Rishbeth \& Williams, $1985)$ radar system is a combination of three active radars operating at 224, 500, and $931 \mathrm{MHz}$ located on Svalbard, Northern Scandanavia, also providing routine ionospheric studies (a new system known as EISCAT_3D is under development, aiming to provide $3 \mathrm{D}$ monitoring of the atmosphere and ionosphere for space weather science and operations). As well as these radar systems there are hundreds of ionosonde instruments operating globally. They are grouped into several networks of instruments such as Low-latitude Ionospheric Sensor Network (LISN) and Realistic Ionosphere (RI) networks, which participate in the United Nations International Space Weather Initiative (ISWI). Because the individual instruments number in the hundreds, only the general frequency of operation from $1 \mathrm{MHz}$ to
$20 \mathrm{MHz}$ is indicated by the "Ionosonde*" marker in Figure 4. To add to this, there are thousands of Global Navigation Satellite System (GNSS) monitors that observe ionospheric scintillation at 1.23 and $\sim 1.58 \mathrm{GHz}$ (in L-band). As outlined in Section 5.2, ionospheric observations are among the most popular space weather data products and LOFAR4SW will be one of the most sensitive and advanced amongst the stations providing textbfthis kind of data.

LOFAR4SW will also provide complimentary observations of SRBs via both imaging spectroscopy and tied-array imaging. Ground-based SRB monitors number in the hundreds worldwide. They range in complexity from interferometers to single dish/ antennas and receivers. Some of the most notable radio burst monitors used in space weather science and operations are the multiple sites of RSTN (Guidice, 1979), providing $24 \mathrm{~h}$ dynamic spectra observations from four separate sites around the world; the Nançay Decametric Array (NDA; Lecacheux, 2000) operating from $10 \mathrm{MHz}$ to $80 \mathrm{MHz}$ and the "Observation Radiospectrographique pour FEDOME et l'Étude des Éruptions Solaires" (ORFÉES; https://www.obs-nancay.fr) observing 
from $100 \mathrm{MHz}$ to $1000 \mathrm{MHz}$, operated at the Nançay Radioastronomy Facility in central France; the eCALLISTO network (Benz et al., 2009) with several sites worldwide operating anywhere between $45 \mathrm{MHz}$ and $870 \mathrm{MHz}$ (depending on the site), also providing $24 \mathrm{~h}$ monitoring of SRBs. These are just some of the many individual stations and networks of SRB monitors worldwide, so only the general frequency of operation is indicated as "SRB Monitoring*" in Figure 4. Importantly, LOFAR4SW's lowest frequencies of operation extend down to the ionospheric cut-off at 10-20 MHz, joining the highest frequency of operation of the small number of space-borne SRB monitoring platforms, namely WIND/WAVES (Bougeret et al., 1995), STEREO/WAVES (Bougeret et al., 2008), Parker Solar Probe (PSP) FIELDS (Bale et al., 2016) and Solar Orbiter (SO) Radio and Plasma Waves (RPW; Maksimovic et al., 2007) instruments. These instruments operate from a few $\mathrm{kHz}$ to $\sim 16 \mathrm{MHz}$ and provide observation of interplanetary type II and III bursts (among many other phenomena), which are essential for monitoring interplanetary shock and energetic electron propagation. LOFAR4SW, WAVES and S/WAVES could provide continuous coverage of SRBs from low corona $\left(\sim 2 R_{\odot}\right)$ to interplanetary space $\left(>100 R_{\odot}\right)$, provided they are all functioning simultaneously in the future. The PSP and SO missions will perform several perihelia of the Sun, providing intermittent observations of the inner-heliosphere and solar corona with nominal mission lifetimes up to 2025 and 2027, respectively. LOFAR4SW therefore has the opportunity to overlap with these new and future missions, potentially providing simultaneous radio (as well as in-situ) observations from inner-heliosphere and Earth-based platforms.

Overall, Figure 4 shows where LOFAR4SW sits in comparison to the major radio research facilities in space weather, bridging the gap between solar, heliospheric, and ionospheric observations, as well as having the capability of multiple observing techniques such as imaging spectroscopy, tied-array imaging and general beam-forming. LOFAR4SW will therefore be one of the most versatile and advanced instruments in use for space weather science and operations. The characteristics of the facilities mentioned above is provided in Table 1.

\subsection{Space weather service providers utilising radio observations}

Here we define space weather service providers as any institute or body that uses the data/metadata/analysis from space weather instruments to provide forecasts and warnings of space weather phenomena. The dissemination of data and forecasts are either via a website or an email warning system. As mentioned, service providers are the main stakeholder of information from instruments and observatories. Their primary goal is to act as liaison between scientists, who record and analyse data, and industry or government bodies who use the results of these analyses.

There are 22 space weather service providers to monitor solar, heliospheric, and/or ionospheric activity. Table 2 lists each SW service provider and the radio data used in their service provision. The most popular space weather radio disseminated through the SW service providers is ionospheric data, with 19 out of 22 using these data. This is followed by radio flux monitoring at single frequencies such as F10.7 (9/22), SRB monitoring (10/22), and finally observations of
Table 2. Worldwide space weather service providers and associated radio data provision on their public domain websites.

\begin{tabular}{|c|c|c|c|c|}
\hline SW service provider & Ionosphere & SRBs & IPS & F10.7 \\
\hline SWPC & レ & レ & レ & レ \\
\hline MOSWOC & 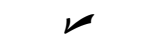 & $\swarrow$ & $\swarrow$ & $\nu$ \\
\hline KSWC & レ & レ & レ & レ \\
\hline SCiSMEX & レ & レ & 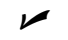 & レ \\
\hline SIDC & 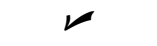 & レ & レ & \\
\hline SRC-RWC & レ & レ & 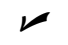 & \\
\hline SWS & & レ & & \\
\hline NISR & $\nu$ & $\nu$ & & \\
\hline IPG & $\swarrow$ & レ & & $\nu$ \\
\hline SEPC & レ & & & $\nu$ \\
\hline KNMI & レ & & & レ \\
\hline SWACI & レ & & & \\
\hline NPL-RWC & レ & & & \\
\hline SWIFtS & 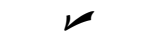 & & & \\
\hline SWFC & レ & & & \\
\hline SANSA & $\swarrow$ & & & \\
\hline LSWC & レ & & & \\
\hline deSWua & $\swarrow$ & & & \\
\hline IONOCY & レ & & & \\
\hline CSWFC & & & & レ \\
\hline SRC-RWC & & & & $\nu$ \\
\hline ESC & & レ & & \\
\hline
\end{tabular}

SWPC, Space Weather Prediction Centre (USA); MOSWOC, Met Office Space Weather Operations Centre (UK); KSWC, Korean SpaceWeather Centre (Republic of Korea); SCiSMEX, SpaceWeather Service Mexico (Mexico); SIDC, Solar Influences Data Analysis Center (Belgium); SRC-RWC, Space Research CenterRegional Warning Center (Poland); SWS, Australian Bureau of Meteorology Space Weather Services (Australia); NISR, National Institute for Space Research (Brazil); IPG, Institute of Applied Geophysics (Russia); SEPC, Space Environment Prediction Center (China); KNMI, Royal Netherlands Meteorological Institute (Nethlerlands); SWACI, Space Weather Application Centre (Germany); NPL-RWC, National Physical Laboratory-Regional Warning Center (India); SWIFtS, Space Weather Information and Forecast Services (Indonesia); SWFC, Space Weather Forecast Center (Japan); SANSA, South African National Space Agency (South Africa); LSWC, Lund Space Weather Centre (Sweden); ESWUA, Electronic Space Weather Upper Atmosphere site (Italy); IONOCY, Cyprus Ionospheric Research Group (Cyprus); CSWFC, Canadian Space Weather Forecasts Centre (Canada); IAP-RWC, Institute of Atmospheric Physics-Regional Warning Center (Czech Republic); ESC, Expert Services Centre for Heliospheric Weather (Austria).

IPS $(5 / 22)$. This distribution of radio data service provision is not surprising, given that ionospheric data is the most accessible and directly applicable to space weather impact (from nowcasting of radio communication efficiency). Only a small number of service providers use IPS data, most likely due to these kinds of observations being highly specialised and also being in their operational infancy. As the technique becomes more established it is likely that more service providers will use it as a standard, given it is now one of the primary and few methods of observing CMEs and solar wind transients in the heliosphere.

Figure 5 shows the worldwide distribution of space weather services along with some of the primary radio instruments in use today. LOFAR4SW holds a strategic position, as it will be a key IPS station at European and African longitudes and 
has good proximity to several space weather service providers concentrated in Europe. As such, LOFAR4SW will be an integral part of the European space weather research and operations infrastructure.

Many of the institutes described above act in close collaboration as part of a global space weather forecasting effort, and they are generally members of various consortia including the International Space Environmental Service (ISES), the PanEuropean Consortium for Aviation Space Weather User Services (PECASUS), the consortium of Australia, Canada, France, and Japan (ACFJ), the European Space Agency (ESA) Space Situational Awareness (SSA) programme, the International Civil Aviation Organization (ICAO) and as part of the World Meteorological Organisation (WMO). This again reflects the worldwide integration of space weather service provision, with radio data provision being an integral part of this global service.

\section{Summary}

In this paper we reviewed the space weather phenomena observable in the radio domain, the space weather indices to which radio observations contribute, and the techniques that are employed at radio wavelengths in the observation of space weather phenomena. Solar flares, CMEs, CIRs, and SEPs each have at least one signature in the radio domain and the techniques used to observe these phenomena range from basic time series to imaging spectroscopy. The instruments that employ such techniques for either space weather science or operations number in the hundreds and we have provided an overview of the existing, and planned, radio instrumentation operating in this domain. It is by no means exhaustive, but attempts to summarise the most commonly used instrumentation providing observations for space weather and the techniques they most commonly employ.

The overview of space weather phenomena, instrumentation and techniques was followed by a survey of the radio data provided by the primary space weather service providers. Having surveyed the existing providers and the radio data they use, we find that their radio data provision is highly varied. While some services concentrate solely on ionospheric observations, others provide data and forecasts of almost all available radio data operating in a space weather capacity. We find that 19/22 provide ionospheric data, 10/22 provide SRB monitoring, 9/22 provide radio flux monitoring (F10.7), and 5/22 IPS monitoring. IPS monitoring has the potential to become an integral part of any space weather service provision. Including the developments in Faraday rotation observations, such a technique may be one of the few ways to predict the interplanetary magnetic field strength and direction. However this science is still in its infancy, which may explain its lack of adoption as an operational space weather technique.

Overall, this work has shown radio data forms an integral part of the infrastructure surrounding space weather observations, science, and operations. Amongst the multiple radio instruments operating in a space weather capacity today, LOFAR4SW will hold a special position as one of the most sensitive and versatile. It will have the ability to observe the Sun, heliosphere, and ionosphere routinely and simultaneously, while using a variety of advanced techniques only available to instruments of its kind i.e. phased-array interferometers capable of performing imaging spectroscopy, as well as tied-array imaging and general beam-forming. It will therefore be an integral part of the space weather research and operations infrastructure in the near future.

Acknowledgements. This work was supported by the European Commission Horizon 2020 INFRADEV-1-2017 LOFAR4SW project number 777442 . We would like to thank Joris Verbiest for some useful comments on the writing of the article. The editor thanks J. Americo Gonzalez-Esparza and Dale Gary for their assistance in evaluating this paper.

\section{References}

Acebal AO, Sojka JJ. 2011. A flare sensitive $3 \mathrm{~h}$ solar flux radio index for space weather applications. Space Weather 9: S07004. https://doi.org/10.1029/2010SW000585.

Agueda N, Lario D. 2016. Release history and transport parameters of relativistic solar electrons inferred from near-the-sun in situ observations. Astrophys J 829: 131. https://doi.org/10.3847/0004637X/829/2/131.

Agueda N, Klein K-L, Vilmer N, Rodrguez-Gasén R, Malandraki OE, et al. 2014. Release timescales of solar energetic particles in the low corona. Astron Astrophys 570: A5. https://doi.org/10.1051/ 0004-6361/201423549.

Anastasiadis A, Papaioannou A, Sandberg I, Georgoulis M, Tziotziou K, Kouloumvakos A, Jiggens P. 2017. Predicting flares and solar energetic particle events: The FORSPEF tool. Sol Phys 292: 134. https://doi.org/10.1007/s11207-017-1163-7.

Asai K, Ishida Y, Kojma M, Maruyama K, Misawa H, Yoshimi N. 1995. Multi-station system for solar wind observations using the interplanetary scintillation method. J Geomagn Geoelectr 47(11): 1107-1112. https://doi.org/10.5636/jgg.47.1107.

Bain HM, Krucker S, Glesener L, Lin RP. 2012. Radio imaging of shock-accelerated electrons associated with an erupting plasmoid on 2010 November 3. Astrophys J 750: 44. https://doi.org/ 10.1088/0004-637X/750/1/44.

Bain HM, Krucker S, Saint-Hilaire P, Raftery CL. 2014. Radio imaging of a type IVM radio burst on the 14th of August 2010. Astrophys J 782: 43. https://doi.org/10.1088/0004-637X/782/1/43.

Balch CC. 1999. SEC proton prediction model: verification and analysis. Rad Meas 30: 231-250. https://doi.org/10.1016/S13504487(99)00052-9.

Bale SD, Goetz K, Harvey PR, Turin P, Bonnell JW, et al. 2016. The FIELDS instrument suite for solar probe plus. Measuring the coronal plasma and magnetic field, plasma waves and turbulence, and radio signatures of solar transients. Space Sci Rev 204: 49-82. https://doi.org/10.1007/s11214-016-0244-5.

Bastian TS, Pick M, Kerdraon A, Maia D, Vourlidas A. 2001. The coronal mass ejection of 1998 April 20: Direct imaging at radio wavelengths. Astrophys J Lett 558: L65-L69. https://doi.org/ 10.1086/323421.

Benz AO, Monstein C, Meyer H, Manoharan PK, Ramesh R, Altyntsev A, Lara A, Paez J, Cho K-S. 2009. A world-wide net of solar radio spectrometers: e-CALLISTO. Earth Moon Planets 104: 277-285. https://doi.org/10.1007/s11038-008-9267-6.

Bilitza D, Altadill D, Zhang Y, Mertens C, Truhlik V, Richards P, McKinnell L-A, Reinisch B. 2014. The international reference ionosphere 2012 - a model of international collaboration. J Space Weather Space Clim 4: A07. https://doi.org/10.1051/swsc/2014004. 
Bisi M, Fallows R, Breen A, ONeill I. 2010a. Interplanetary scintillation observations of stream interaction regions in the solar wind. Sol Phys 261(1): 149-172.

Bisi MM, Breen AR, Jackson BV, Fallows RA, Walsh AP, et al. 2010b. From the sun to the earth: The 13 May 2005 coronal mass ejection. Sol Phys 265: 49-127. https://doi.org/10.1007/s11207010-9602-8.

Bisi MM, Americo Gonzalez-Esparza J, Jackson B, AguilarRodriguez E, Tokumaru M, et al. 2017. The worldwide interplanetary scintillation (IPS) stations (WIPSS) network in support of space-weather science and forecasting. In: EGU General Assembly Conference Abstracts, 23-28 April, Vienna, Austria, Vol. 19, p. 13454.

Boteler DH, Pirjola RJ, Nevanlinna H. 1998. The effects of geomagnetic disturbances on electrical systems at the earth's surface. Adv Space Res 22: 17-27. https://doi.org/10.1016/S02731177(97)01096-X.

Bougeret J-L, Kaiser ML, Kellogg PJ, Manning R, Goetz K, et al. 1995. Waves: The radio and plasma wave investigation on the wind spacecraft. Space Sci Rev 71: 231-263. https://doi.org/ 10.1007/BF00751331.

Bougeret JL, Goetz K, Kaiser ML, Bale SD, Kellogg PJ, et al. 2008. S/WAVES: The radio and plasma wave investigation on the STEREO mission. Space Sci Rev 136: 487-528. https://doi.org/ 10.1007/s11214-007-9298-8.

Carley EP, Long DM, Byrne JP, Zucca P, Bloomfield DS, McCauley J, Gallagher PT. 2013. Quasiperiodic acceleration of electrons by a plasmoid-driven shock in the solar atmosphere. Nat Phys 9: 811816. https://doi.org/10.1038/nphys2767.

Carley EP, Vilmer N, Simões PJA, Fearraigh BÓ. 2017. Estimation of a coronal mass ejection magnetic field strength using radio observations of gyrosynchrotron radiation. A\&A 608: A137. https://doi.org/10.1051/0004-6361/201731368.

Cerruti AP, Kintner PM, Gary DE, Lanzerotti LJ, de Paula ER, Vo HB. 2006. Observed solar radio burst effects on GPS/Wide Area Augmentation System carrier-to-noise ratio. Space Weather 4: S10006. https://doi.org/10.1029/2006SW000254.

Cerruti AP, Kintner PM, Gary DE, Mannucci AJ, Meyer RF, Doherty P, Coster AJ. 2008. Effect of intense December 2006 solar radio bursts on GPS receivers. Space Weather 6: S10D07. https://doi.org/10.1029/2007SW000375.

Cranmer SR. 2009. Coronal Holes. Liv Rev Sol Phys 6(1): 3. https://doi.org/10.12942/lrsp-2009-3.

Cremades H, Iglesias FA, St. Cyr OC, Xie H, Kaiser ML, Gopalswamy N. 2015. Low-frequency type-II radio detections and coronagraph data employed to describe and forecast the propagation of 71 CMEs/shocks. Sol Phys 290: 2455-2478. https://doi.org/10.1007/s11207-015-0776-y.

Cucinotta FA, Hu S, Schwadron NA, Kozarev K, Townsend LW, Kim M-HY. 2010. Space radiation risk limits and Earth-MoonMars environmental models. Space Weather 8: S00E09. https://doi.org/10.1029/2010SW000572.

Dagkesamanskii RD. 2009. The Pushchino Radio Astronomy Observatory of the P N Lebedev Physical Institute Astro Space Center: Yesterday, today, and tomorrow. Phys Uspekhi 52(11): 1159. http://stacks.iop.org/1063-7869/52/i=11/a=R09.

Dauphin C, Vilmer N, Krucker S. 2006. Observations of a soft X-ray rising loop associated with a type II burst and a coronal mass ejection in the 03 November 2003 X-ray flare. A\&A 455: 339-348. https://doi.org/10.1051/0004-6361:20054535.

Denardini CM, Dasso S, Gonzalez-Esparza JA. 2016. Review on space weather in Latin America. 2. The research networks ready for space weather. Adv Space Res 58(10): 1940-1959. Space and
Geophysical Research related to Latin America - Part 2, https://doi.org/10.1016/j.asr.2016.03.013.

Dresing N, Gómez-Herrero R, Heber B, Klassen A, Temmer M, Veronig A. 2018. Long-lasting injection of solar energetic electrons into the heliosphere. $A \& A$ 613: A21. https://doi.org/ 10.1051/0004-6361/201731573.

Dulk GA, Marsh KA. 1982. Simplified expressions for the gyrosynchrotron radiation from mildly relativistic, nonthermal and thermal electrons. Astrophys J 259: 350-358. https://doi.org/ 10.1086/160171.

Fallows RA, Asgekar A, Bisi MM, Breen AR, ter-Veen S. 2013. The dynamic spectrum of interplanetary scintillation: First solar wind observations on LOFAR. Sol Phys 285: 127-139. https://doi.org/ 10.1007/s11207-012-9989-5.

Fallows RA, Coles WA, McKay-Bukowski D, Vierinen J, Virtanen II, et al. 2014. Broadband meter-wavelength observations of ionospheric scintillation. J Geophys Res (Space Phys) 119: 10. https://doi.org/10.1002/2014JA020406.

Fallows RA, Bisi MM, Forte B, Ulich T, Konovalenko AA, Mann G, Vocks C. 2016. Separating nightside interplanetary and ionospheric scintillation with LOFAR. Astrophys J Lett 828: L7. https://doi.org/10.3847/2041-8205/828/1/L7.

Fleishman GD, Nita GM, Kontar EP, Gary DE. 2016. Narrowband gyrosynchrotron bursts: Probing electron acceleration in solar flares. Astrophys J 826: 38. https://doi.org/10.3847/0004-637X/ $826 / 1 / 38$.

Gary DE. 2016. Role of solar radio observations in space weather. In: 2016 URSI Asia-Pacific Radio Science Conference (URSI AP-RASC), 21-25 August, Seoul, South Korea, pp. 1-4. https://doi.org/10.1109/URSIAP-RASC.2016.7883552.

Glover A, Hilgers A, Rosenqvist L, Bourdarie S. 2008. Interplanetary proton cumulated fluence model update. Adv Space Res 42: 1564 1568. https://doi.org/10.1016/j.asr.2007.08.023.

Grechnev VV, Lesovoi SV, Smolkov GY, Krissinel BB, Zandanov VG, et al. 2003. The Siberian Solar Radio Telescope: The current state of the instrument, observations, and data. Sol Phys 216: 239 272. https://doi.org/10.1023/A:1026153410061.

Guidice DA. 1979. Sagamore Hill Radio Observatory, Air Force Geophysics Laboratory, Hanscom Air Force Base, Massachusetts 01731. Report. In: Bulletin of the American Astronomical Society, Vol. 11, American Astronomical Society, pp. 311-312.

Jackson B, Clover J, Hick P, Buffington A, Bisi M, Tokumaru M. 2013. Inclusion of real-time in-situ measurements into the UCSD time-dependent tomography and its use as a forecast algorithm. Sol Phys 285(1-2): 151-165.

Jackson BV, Odstrcil D, Yu H-S, Hick PP, Buffington A, et al. 2015. The UCSD kinematic IPS solar wind boundary and its use in the ENLIL 3-D MHD prediction model. Space Weather 13: 104-115. https://doi.org/10.1002/2014SW001130.

Jensen EA, Hick PP, Bisi MM, Jackson BV, Clover J, Mulligan T. 2010. Faraday rotation response to coronal mass ejection structure. Sol Phys 265: 31-48. https://doi.org/10.1007/ s11207-010-9543-2.

Kahler SW. 2007. Solar sources of heliospheric energetic electron events - shocks or flares? Space Sci Rev 129: 359-390. https://doi. org/10.1007/s11214-007-9143-0.

Kappenman JG. 1996. Geomagnetic Storms and Their Impact on Power Systems. IEEE Power Eng Rev 16(5): 5-. https://doi.org/ 10.1109/MPER.1996.491910.

Kerdraon A, Delouis J-M. 1997. The Nançay Radioheliograph. In: Coronal Physics from Radio and Space Observations, Vol. 483 of Lecture Notes in Physics, Trottet G (Ed.), Springer Verlag, Berlin, pp. 192. https://doi.org/10.1007/BFb0106458 
Klein K-L, Trottet G, Klassen A. 2010. Energetic particle acceleration and propagation in strong CME-less flares. Sol Phys 263: 185-208. https://doi.org/10.1007/s11207-010-9540-5.

Klein K-L, Matamoros CS, Zucca P. 2018. Solar radio bursts as a tool for space weather forecasting. C R Phys 19: 36-42. https://doi. org/10.1016/j.crhy.2018.01.005.

Kontar EP, Yu S, Kuznetsov AA, Emslie AG, Alcock B, Jeffrey NLS, Melnik VN, Bian NH, Subramanian P. 2017. Imaging spectroscopy of solar radio burst fine structures. Nat Commun 8: 1515. https://doi.org/10.1038/s41467-017-01307-8.

Kooi JE, Fischer PD, Buffo JJ, Spangler SR. 2017. VLA measurements of Faraday rotation through coronal mass ejections. Sol Phys 292: 56. https://doi.org/10.1007/s11207-017-1074-7.

Kouloumvakos A, Nindos A, Valtonen E, Alissandrakis CE, Malandraki O, Tsitsipis P, Kontogeorgos A, Moussas X, Hillaris A. 2015. Properties of solar energetic particle events inferred from their associated radio emission. $A \& A$ 580: A80. https://doi.org/ 10.1051/0004-6361/201424397.

Kuroda N, Gary DE, Wang H, Fleishman GD, Nita GM, Jing J. 2018. Three-dimensional forward-fit modeling of the hard X-ray and microwave emissions of the 2015 June 22 M6.5 Flare. Astrophys J 852: 32. https://doi.org/10.3847/1538-4357/aa9d98.

Laitinen T, Effenberger F, Kopp A, Dalla S. 2018. The effect of turbulence strength on meandering field lines and solar energetic particle event extents. J Space Weather Space Clim 8(27): A13. https://doi.org/10.1051/swsc/2018001.

Lario D, Kwon R-Y, Riley P, Raouafi NE. 2017. On the Link between the release of solar energetic particles measured at widespread heliolongitudes and the properties of the associated coronal shocks. Astrophys J 847: 103. https://doi.org/10.3847/1538-4357/aa89e3.

Lecacheux A. 2000. The Nançay Decameter Array: A useful step towards giant, new generation radio telescopes for long wavelength radio astronomy. Washington DC Am Geophys Union Geophys Monogr Ser 119: 321. https://doi.org/10.1029/GM119p0321.

Magdalenić J, Marqué C, Krupar V, Mierla M, Zhukov AN, Rodriguez L, Maksimović M, Cecconi B. 2014. Tracking the CME-driven shock wave on 2012 March 5 and radio triangulation of associated radio emission. Astrophys J 791: 115. http://doi.org/ 10.1088/0004-637X/791/2/115.

Maia DJF, Gama R, Mercier C, Pick M, Kerdraon A, Karlický M. 2007. The radio-coronal mass ejection event on 2001 April 15. Astrophys J 660: 874-881. https://doi.org/10.1086/508011.

Maksimovic M, Bale SD, Vaivads A, Krasnoselskikh V, Chust T, et al. 2007. A radio and plasma wave experiment for the solar orbiter mission. In: Second Solar Orbiter Workshop, Vol. 641 of ESA Special Publication, p. 38.

Marqué C, Klein K-L, Monstein C, Opgenoorth H, Pulkkinen A, Buchert S, Krucker S, Van Hoof R, Thulesen P. 2018. Solar radio emission as a disturbance of aeronautical radionavigation. $J$ Space Weather Space Clim 8(27): A42. https://doi.org/10.1051/swsc/ 2018029.

McKay-Bukowski D, Vierinen J, Virtanen II, Fallows R, Postila M, et al. 2015. KAIRA: The Kilpisjärvi Atmospheric Imaging Receiver Array - system overview and first results. IEEE Trans Geosci Remote Sens 53: 1440-1451. https://doi.org/10.1109/ TGRS.2014.2342252.

Mei Y, Wang F, Wang W, Chen L, Liu Y, Deng H, Dai W, Liu C, Yan Y. 2018. GPU-based high-performance imaging for Mingantu spectral radioheliograph. PASP 130(1): 014503. https://doi.org/ 10.1088/1538-3873/aa9608.

Mejia-Ambriz JC, Villanueva-Hernandez P, Gonzalez-Esparza JA, Aguilar-Rodriguez E, Jeyakumar S. 2010. Observations of interplanetary scintillation (IPS) using the Mexican Array Radio
Telescope (MEXART). Sol Phys 265: 309-320. https://doi.org/ 10.1007/s11207-010-9562-z.

Melrose DB. 2017. Coherent emission mechanisms in astrophysical plasmas. Rev Mod Plasma Phys 1: 5. https://doi.org/10.1007/ s41614-017-0007-0.

Morosan DE, Gallagher PT, Zucca P, Fallows R, Carley EP, et al. 2014. LOFAR tied-array imaging of type III solar radio bursts. A\&A 568: A67. https://doi.org/10.1051/0004-6361/201423936.

Morosan DE, Gallagher PT, Zucca P, O'Flannagain A, Fallows R, et al. 2015. LOFAR tied-array imaging and spectroscopy of solar $\mathrm{S}$ bursts. A\&A 580: A65. https://doi.org/10.1051/0004-6361/ 201526064.

Muhammad B, Alberti V, Marassi A, Cianca E, Messerotti M. 2015. Performance assessment of GPS receivers during the September 24, 2011 solar radio burst event. J Space Weather Space Clim 5 (27): A32. https://doi.org/10.1051/swsc/2015034.

Nakajima H, Nishio M, Enome S, Shibasaki K, Takano T, et al. 1994. The Nobeyama radioheliograph. IEEE Proc 82: 705-713.

Nelson GJ, Melrose DB. 1985. Type II bursts, CSIRO, pp. 333-359.

Odstrcil D. 2003. Modeling 3-D solar wind structure. Adv Space Res 32: 497-506. https://doi.org/10.1016/S0273-1177(03)00332-6.

Patil AH, Yatawatta S, Koopmans LVE, de Bruyn AG, Brentjens MA, et al. 2017. Upper limits on the $21 \mathrm{~cm}$ epoch of reionization power spectrum from one night with LOFAR. Astrophys $J$ 838: 65 . https://doi.org/10.3847/1538-4357/aa63e7.

Pick M, Vilmer N. 2008. Sixty-five years of solar radioastronomy: Flares, coronal mass ejections and Sun Earth connection. Astron Astrophys Rev 16: 1-153. https://doi.org/10.1007/s00159-0080013-x.

Pick M, Stenborg G, Démoulin P, Zucca P, Lecacheux A. 2016. Homologous solar events on 2011 January 27: Build-up and propagation in a complex coronal environment. Astrophys $J$ 823: 5. https://doi.org/10.3847/0004-637X/823/1/5.

Prikryl P, Jayachandran PT, Mushini SC, Richardson IG. 2012. Toward the probabilistic forecasting of high-latitude GPS phase scintillation. Space Weather 10: S08005. https://doi.org/10.1029/ 2012SW000800.

Ramesh R, Subramanian KR, Sundararajan MS, Sastry CV. 1998. The Gauribidanur Radioheliograph. Sol Phys 181(2): 439-453. https://doi.org/10.1023/A:1005075003370.

Reames DV. 1999. Particle acceleration at the Sun and in the heliosphere. Space Sci Rev 90: 413-491. https://doi.org/10.1023/ A:1005105831781.

Reames DV. 2013. The two sources of solar energetic particles. Space Sci Rev 175(1-4): 53-92. https://doi.org/10.1007/s11214013-9958-9.

Reid HAS, Kontar EP. 2017. Imaging spectroscopy of type U and J solar radio bursts with LOFAR. A\&A 606: A141. https://doi.org/ 10.1051/0004-6361/201730701.

Reid HAS, Kontar EP. 2018. Solar type III radio burst time characteristics at LOFAR frequencies and the implications for electron beam transport. $A \& A$ 614: A69. https://doi.org/10.1051/ 0004-6361/201732298.

Richardson IG. 2018. Solar wind stream interaction regions throughout the heliosphere. Liv Rev Sol Phys 15: 1. https://doi. org/10.1007/s41116-017-0011-z.

Rishbeth H, Williams PJS. 1985. The EISCAT ionospheric radar the system and its early results. Astron Soc 26: 478-512.

Selvakumaran R, Maurya AK, Gokani SA, Veenadhari B, Kumar S, Venkatesham K, Phanikumar DV, Singh AK, Siingh D, Singh R. 2015. Solar flares induced D-region ionospheric and geomagnetic perturbations. J Atmos Sol-Terr Phys 123: 102-112. https://doi. org/10.1016/j.jastp.2014.12.009. 
Shimwell TW, Röttgering HJA, Best PN, Williams WL, Dijkema TJ, et al. 2017. The LOFAR two-metre sky survey. I. Survey description and preliminary data release. $A \& A$ 598: A104. https://doi.org/10.1051/0004-6361/201629313.

Stappers BW, Hessels JWT, Alexov A, Anderson K, Coenen T, et al. 2011. Observing pulsars and fast transients with LOFAR. $A \& A$ 530: A80. https://doi.org/10.1051/0004-6361/201116681.

Sukumar S, Velusamy T, Pramesh Rao A, Swarup G, Bagri DS. 1988. Ooty synthesis radio telescope - Design and performance. Bull Astron Soc India 16: 93-110.

Tiburzi C, Verbiest JPW, Shaifullah GM, Janssen GH, Anderson JM, et al. 2019. On the usefulness of existing solar wind models for pulsar timing corrections. Mon Not R Astron Soc 487: 394-408. https://doi.org/10.1093/mnras/stz1278.

Trichtchenko L, Boteler DH. 2001. Specification of geomagnetically induced electric fields and currents in pipelines. $J$ Geophys Res 106: 21039-21048. https://doi.org/10.1029/2000JA000207.

Tsurutani BT, Gonzalez WD, Gonzalez ALC, Guarnieri FL, Gopalswamy N, et al. 2006. Corotating solar wind streams and recurrent geomagnetic activity: A review. J Geophys Res (Space Phys) 111: A07S01. https://doi.org/10.1029/2005JA011273.

Tsurutani BT, Verkhoglyadova OP, Mannucci AJ, Lakhina GS, Li G, Zank GP. 2009. A brief review of solar flare effects on the ionosphere. Radio Sci 44: RS0A17. https://doi.org/10.1029/2008RS004029.

van de Kamp M, Cannon PS, Terkildsen M. 2009. Effect of the ionosphere on defocusing of space-based radars. Radio Sci 44: RS1003. https://doi.org/10.1029/2007RS003808.

van Haarlem MP, Wise MW, Gunst AW, Heald G, McKean JP, et al. 2013. LOFAR: The LOw-Frequency ARray. A\&A 556: A2. https://doi.org/10.1051/0004-6361/201220873.

Vilà-Valls J, Closas P, Curran JT. 2017. Multi-frequency GNSS robust carrier tracking for ionospheric scintillation mitigation. $J$ Space Weather Space Clim 7(27): A26. https://doi.org/10.1051/ swsc/2017020.

Vocks C, Mann G, Breitling F, Bisi MM, Daôbrowski B, et al. 2018. LOFAR observations of the quiet solar corona. A\&A 614: A54. https://doi.org/10.1051/0004-6361/201630067.

Wang C. 2010. New chains of space weather monitoring stations in China. Space Weather 8: S08001. https://doi.org/10.1029/ 2010SW000603.

Warmuth A, Mann G. 2004. The application of radio diagnostics to the study of the solar drivers of space weather. In: Lecture Notes in Physics, Berlin Springer Verlag, Vol. 656, Scherer K, Fichter H, Herber B (Ed.), Springer Verlag, Berlin, p. 49. https://doi.org/ 10.1007/978-3-540-31534-6-3.

Wei LH, Homeier N, Gannon JL. 2013. Surface electric fields for North America during historical geomagnetic storms. Space Weather 11: 451-462. https://doi.org/10.1002/swe.20073.

White SM, Benz AO, Christe S, Fárnk F, Kundu MR, et al. 2011. The relationship between solar radio and hard X-ray emission. Space Sci Rev 159(1-4): 225-261. https://doi.org/10.1007/s11214010-9708-1.

Wild JP. 1950. Observations of the Spectrum of High-Intensity Solar Radiation at Metre Wavelengths. III. Isolated Bursts. Aust J Sci Res A Phys Sci 3: 541.

Wild JP, Sheridan KV, Trent GH. 1959. The transverse motions of the sources of solar radio bursts. In: URSI Symp. 1: Paris Symposium on Radio Astronomy, Vol. 9 of IAU Symposium, Bracewell RN (Ed.), Stanford University Press, Stanford, CA, p. 176.

Xapsos MA, Stauffer C, Jordan T, Barth JL, Mewaldt RA. 2007. Model for cumulative solar heavy ion energy and linear energy transfer spectra. IEEE Trans Nucl Sci 54(6): 1985-1989. https://doi.org/10.1109/TNS.2007.910850.
Yasyukevich Y, Astafyeva E, Padokhin A, Ivanova V, Syrovatskii S, Podlesnyi A. 2018. The 6 September 2017 XClass solar flares and their impacts on the ionosphere, GNSS, and HF radio wave propagation. Space Weather 16: 1013-1027. https://doi.org/ 10.1029/2018SW001932.

Yu H-S, Jackson BV, Hick PP, Buffington A, Odstrcil D, Wu C-C, Davies JA, Bisi MM, Tokumaru M. 2015. 3D reconstruction of interplanetary scintillation (IPS) remote-sensing data: Global solar wind boundaries for driving 3D-MHD models. Sol Phys 290: 2519-2538. https://doi.org/10.1007/s11207-015-0685-0.

Žigman V, Grubor D, Šulić D. 2007. D-region electron density evaluated from VLF amplitude time delay during X-ray solar flares. J Atmos Sol-Terr Phys 69: 775-792. https://doi.org/ 10.1016/j.jastp.2007.01.012.

Zucca P, Carley EP, McCauley J, Gallagher PT, Monstein C, McAteer RTJ. 2012. Observations of low frequency solar radio bursts from the Rosse solar-terrestrial observatory. Sol Phys 280: 591-602. https://doi.org/10.1007/s11207-012-9992-x.

Zucca P, Núñez M, Klein K. 2017. Exploring the potential of microwave diagnostics in SEP forecasting: The occurrence of SEP events. J Space Weather Space Clim 7(27): A13. https://doi.org/ $10.1051 / \mathrm{swsc} / 2017011$.

\section{Appendix}

\section{The LOFAR4SW project}

LOFAR is not currently a space weather-dedicated facility. The aim of the LOFAR4SW project is to design a system upgrade such that the instrument can perform space weather observations, including of the Sun, heliosphere, and Earth's ionosphere, simultaneously with its core radio astronomy remit. This will include designs for a significant upgrade of hardware and software which builds on the technology and infrastructure of the current LOFAR system. Thus the project prepares the ground work for a large-scale high-end research facility in which completely simultaneous and independent observing modes provide continuous access to two research communities: radio astronomy and space weather research.

One of the major hardware upgrades includes a redesign of the analogue beam-forming capabilities of the current LOFAR high band antenna (HBA) tiles. The current system uses a single analogue beam per HBA tile which restricts the field of view of the HBA antennas before any digital beam-forming takes place. The initial analogue beam-forming renders observation of the Sun and heliosphere impossible while in use for another astronomy application. LOFAR4SW is designing a dual beamformer for the HBA tile such that observation of space weather phenomena will always be possible, even while the system is being used for other astronomical observations.

The station back-end is currently being re-developed as part of a separate upgrade effort such that it can receive and digitally beam-form signals from all of the available low band antennas (LBAs) and HBAs simultaneously, which is not a capability of the current system. This upgrade will also significantly increase the digital beamforming capacity at each station, at least doubling the bandwidth which can be sampled from $96 \mathrm{MHz}$ to $192 \mathrm{MHz}$, which can be spread as desired over the observing band. The LOFAR central correlator and network will also be upgraded to reflect the extra data throughput. The LOFAR4SW 
design then incorporates the station back-end extensions of this upgrade necessary to perform the desired space weather observations in parallel. In this regard, the LOFAR4SW system design will also include the necessary dedicated additional networking and central data processing resources to enable space weather observations to run in parallel to astronomy.

The project has already completed its preliminary design review and held several workshops with the international space-weather community to identify the key science requirements of the future system, which in turn are being used to derive the low level technical requirements and develop the system prototypes (e.g., the dual beamformer of the HBA tile). The project is currently mid-way through is lifespan, with expected completion in early 2021. For further details please refer to http://lofar4sw.eu/.

Cite this article as: Carley EP, Baldovin C, Benthem P, Bisi MM, Fallows RA, et al. 2020. Radio observatories and instrumentation used in space weather science and operations. J. Space Weather Space Clim. 10, 7. 